O26:H11 (rfbO26, fliC11); E. coli O111 - to 2 serovars: O111:H8 (5 strains $r f b \mathrm{O} 111, f l i C 8)$ and O111:H2 (12 strains of $r f b \mathrm{O} 111, f l i C 2)$. E. coli $\mathrm{O} 55$ also belonged to 2 serovars: O55:H7 (one strain, $r f b \mathrm{O} 55, \mathrm{fliC} 7$ ) and O55:H6 (five strains of $r f b \mathrm{O} 55$, fliC6). According to our data, 10 strains of $\mathrm{O} 26: \mathrm{H} 11,5$ strains of $\mathrm{O} 111: \mathrm{H} 8$ and 1 strain of $\mathrm{O} 55: \mathrm{H} 7$ had stx 1 gene (encoding the production of shiga-like toxin 1) in combination with eae gene (the adhesion factor, intimin) and could be consider as EHEC. All strains E. coli $\mathrm{O} 111: \mathrm{H} 2$ and $E$. coli $\mathrm{O} 55: \mathrm{H} 6$ and 42 E. coli $\mathrm{O} 26$ : H11 had only eae gene, indicating that these strains belonged to the EPEC.

The introduction of molecular methods of serotyping and detection of virulence factors in laboratory diagnostics makes it possible to confirm the true pathogenicity of $E$. coli strains and to minimize the diagnostic errors in etiological interpretation of acute enteric infections.

\section{COMPARISON OF PHENOTYPIC} AND MOLECULAR-GENETIC PROPERTIES OF THE STRAINS NEISSERIA MENINGITIDIS ISOLATED FROM PATIENTS WITH GENERALIZED FORMS OF MENINGOCOCCAL INFECTION AND CARRIERS

\section{E.A. Martens, S.V. Sidorenko, L.I. Zhelezova}

Pediatric Research and Clinical Center for Infectious Diseases, St. Petersburg, Russia

Characterization of isolates of Neisseria meningitidis obtained from patients with meningococcal disease or from nasopharyngeal swabs of asymptomatic carriers can be achieved by several methods which provide different levels of discrimination.

A total of 42 gram-negative, oxidase-positive diplococcus strains isolated from individuals with meningococcal disease in 2009-2018 years and 65 isolates from 1075 nasopharyngeal carriers in 2016-2018 years were examined by three approaches: serological typing by agglutination, determination of the serogroups by real-time PCR, multilocus sequence typing (MLST). Each strain from patients with meningococcal disease was also determined sensitivity to antibiotics by dilution in broth.

The majority of strains isolated from patients belonged according to the results of agglutination and real-time PCR data to serogroup B (50 and $40.5 \%$ respectively), $\mathrm{C}$ (16.7 and $11.9 \%$ respectively) and $\mathrm{W}$ (14.3\% by results of both methods). Among the isolates from carriers according to the results of agglutination and real-time PCR data were dominated serogroup $\mathrm{W}$ (37.0 and $30.8 \%$ respectively) and B (32.3 and $26.2 \%$ respectively). Invasive isolates of serogroup B were resistant to penicillin (28.6\%), levofloxacin (33.3\%), chloramphenicol $(27.3 \%)$, rifampicin (14.3\%), invasive isolates of serogroup W135 to chloramphenicol $(9.1 \%)$.

MLST established the genetic relationships of the isolates from patients and identified members of known hypervirulent lineage CC11 $(n=4)$.

Six isolates of $N$. meningitidis (invasive and from nasopharyngeal swab) were additionally investigated by whole genome sequencing.

The results are included in the GenBank international database: SRR7352647 SAMN09435696 Nm-146 blood 16-Apr-2018, SRR7352646 SAMN09435695 Nm-105 nasopharyngeal swab Aug-2016 (https://www.ncbi.nlm.nih. gov/sra/SRP150714)

\section{MOLECULAR TYPING IN RESEARCH OF EPIDEMICAL} CHOLERA MANIFESTATION

L.V. Mironova, Zh.Yu. Khunkheeva, A.S. Ponomareva, A.S. Gladkikh, N.O. Bochalgin, E.A. Basov, S.V. Balakhonov Irkutsk Antiplague Research Institute of Rospotrebnadzor, Irkutsk, Russia

The causative agent of El Tor cholera evolved adaptive mechanisms to ensure its' preservation and accumulation in certain ecological niches and provide existence of its population in various climatic and geographical zones. Considering this, epidemics development mechanism differs in endemic and non-endemic areas. Siberian and the Far East regions are non-endemic territories for cholera. The last epidemic complications in this region were reported in 1990s and had a form of certain infection importation cases and acute outbreaks, associated with importations. Along with this, strains of the Vibrio cholerae El Tor, devoid of the pathogenicity determinants, are found annually in environmental objects.

The aim of this work is to elucidate the patterns of cholera epidemiological manifestations in Siberia and the Far East, based on a complex molecular genetic analysis of $V$. cholerae El Tor strains.

In complex assay, using amplification profiling, MLVA-, PFGE-, MLST-, and wgSNP-typing, we found, that $V$. cholerae El Tor strains isolated in epidemic complications, homogenous in basic pathogenicity, pandemicity, persistence determinants along with nucleotide context of housekeeping genes, are characterized by diversity in the associated with pathogenicity genomic loci structure, macrorestriction patterns, SNP-profiles, and structure of variable tandem repeats loci. At the same time, closely related subclones of one clonal variant were identified within the individual outbreaks. Considering the typing data, we can conclude that the outbreaks genesis in the non-endemic territories of the region is determined by the hyperinfectious clone importation. Circulation of closely related subclonal vibrio variants during the outbreak can be caused by environmental factors during the implementation of the water or the contact-household transmission routes.

$V$. cholerae $\mathrm{O} 1 \mathrm{El}$ Tor isolates from surface watercourse in a cholera free period are characterized by a significant polymorphism of MLVA profiles and PFGE genotypes; that indicates a high heterogeneity of the water populations of the microorganism and the probability of microevolutionary changes during persistence in surface watercourse, as well as the possible periodic introduction of new clones into aquatic ecosystems.

Thus, molecular approaches in the analysis of cholera manifestations provides an understanding of the epidemic complications development patterns in the territory and $V$. cholerae persistence in the environment.

\section{PCR FOR DIAGNOSIS OF GONOCOCCAL INFECTION: PANACEA OR ESCAPE FROM REALITY}

N.V. Nikolaeva, A.P. Godovalov, T.I. Karpunina

Acad. E.A. Wagner Perm State Medical University, Perm, Russia

The aim of this study was to provide an analytical assessment of the role and place of PCR in gonococcal infection (GI) monitoring. Clinical guidelines define a set of laboratory tests for its diagnosis, including molecular genetic techniques (MGT). It is considered, that MGT have the highest diagnostic sensitivity, in contrast to the traditional procedures (microscopic, bacteriolo- 